\title{
BMJ Open Angioplasty and stenting for patients with symptomatic intracranial atherosclerosis: study protocol of a randomised controlled trial
}

Xiao-Ping Cui, ${ }^{1}$ Min Lin, ${ }^{1}$ Jun-Shan Mu, ${ }^{1}$ Jian-Xin Ye, ${ }^{1}$ Wen-Qing $\mathrm{He},{ }^{2}$ Mao-Lin Fu, ${ }^{2}$ Hua Li, ${ }^{3}$ Jia-Yang Fang, ${ }^{3}$ Feng-Feng Shen, ${ }^{3}$ Hang Lin ${ }^{1}$

To cite: Cui X-P, Lin M, Mu J-S, et al. Angioplasty and stenting for patients with symptomatic intracranial atherosclerosis: study protocol of a randomised controlled trial. BMJ Open 2016;6:e012175. doi:10.1136/bmjopen-2016012175

- Prepublication history for this paper is available online. To view these files please visit the journal online (http://dx.doi.org/10.1136/ bmjopen-2016-012175).

Received 5 April 2016 Revised 17 September 2016 Accepted 19 September 2016

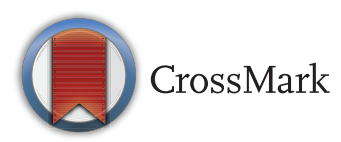

${ }^{1}$ Department of Neurology, Fuzhou General Hospital of Nanjing Command, People's Liberation Army and Clinical Medical College of Fujian Medical University, Fuzhou, China

${ }^{2}$ Department of Neurology, The 180th Hospital of People's Liberation Army, Quanzhou, China

${ }^{3}$ Department of Neurology, The 476th Hospital of People's Liberation Army, Fuzhou, China

Correspondence to

Dr Hang Lin;

hanglindr@126.com

\section{ABSTRACT}

Introduction: Whether adding percutaneous transluminal angioplasty and stenting (PTAS) to background medical treatment is effective for decreasing the incidence of stroke or death in patients with symptomatic intracranial atherosclerosis (ICAS) is still controversial. We perform a randomised controlled trial to examine the effectiveness and safety of an improved PTAS procedure for patients with ICAS. Methods and analysis: A randomised controlled trial will be conducted in three hospitals in China. Eligible patients with ICAS will be randomly assigned to receive medication treatment (MT) plus PTAS or MT alone. The MT will be initiated immediately after randomisation, while the PTAS will be performed when patients report relief of alarm symptoms defined as sudden weakness or numbness. All patients will be followed up at 30 days, 3 and 12 months after randomisation. The primary end point will be the incidence of stroke or death at 30 days after randomisation. Secondary outcomes will be the incidence of ischaemic stroke in the territory of stenosis arteries, the incidence of in-stent restenosis, the Chinese version of the modified Rankin Scale and the Chinese version of the Stroke-Specific Quality of Life (CSQoL).

Ethics and dissemination: The study protocol is approved by institutional review boards in participating hospitals (reference number FZ20160003, 180PLA20160101 and 476PLA2016007). The results of this study will be disseminated to patients, physicians and policymakers through publication in a peer-reviewed journal or presentations in conferences. It is anticipated that the results of this study will improve the quality of the current PTAS procedure and guide clinical decision-making for patients with ICAS. Trial registration number: NCT02689037

\section{BACKGROUND}

Intracranial atherosclerosis (ICAS) is one of the leading causes of cerebral ischaemia. Patients with symptomatic ICAS are at high
Strengths and limitations of this study

- We will use an improved surgical protocol in percutaneous transluminal angioplasty and stenting (PTAS) and exclude patients with perforator strokes for whom the risk of PTAS treatment outweighs the benefit.

- We include hospitals with high-volume enrolment, which reduces the risk of postoperative complications.

- The 1-year incidence of stroke or death after randomisation is not fully studied because the short-term superiority of PTAS over medication treatment has not been confirmed.

risk of recurrent stroke and dementia. ${ }^{1-3} \mathrm{~A}$ stenosis with luminal narrowing over $70 \%$ in a large intracranial artery leads to a $20 \%$ higher risk of subsequent stroke within 1 year, as shown by the Warfarin-Aspirin Symptomatic Intracranial Disease (WASID) trial; even after being treated with aspirin, patients in the WASID trial still showed high incidence of recurrent ischaemic stroke. ${ }^{4}$ The prevalence of ICAS is as high as $46.6 \%$ in the Chinese population, according to the Chinese Intracranial Atherosclerosis trial (CICAS)..$^{5}$ In the CICAS study, patients with ICAS had more severe strokes and longer in-patient stays than those without ICAS. Therefore, effective interventions are needed for preventing strokes in patients with ICAS, especially in those with symptomatic ICAS.

Neurologists usually suggest patients with symptomatic ICAS receive percutaneous transluminal angioplasty and stenting (PTAS) to prevent strokes. ${ }^{6}$ However, the benefit of adding PTAS to medical treatment was not found in the Stenting versus Aggressive Medical Treatment for Intracranial Arterial Stenosis study (SAMMPRIS) ${ }^{6}{ }^{7}$ Whether the result of the SAMMPRIS study was unbiased 
has recently been questioned. ${ }^{8}$ The controversies focus on the inexperience of the operators and the inclusion of patients who were not recommended for PTAS treatment. ${ }^{8-11}$ The question about operators' experience was answered in the subsequent subgroup analysis, which showed that the experience of the operators played little role in the high incidence of postoperative complications, but low-volume enrolment of patients in some of the study sites was an important factor for postoperative complications. ${ }^{12}$ Additionally, vessel size, inclusion of patients with perforator syndrome, and styles of operation procedure were considered as critical factors that increased postoperative complications. ${ }^{8} \quad 11 \quad 13 \quad 14$ The above factors indicate that excluding patients with perforator syndrome or patients with vessel size $\leq 2.5 \mathrm{~mm}$ and including study sites with high-volume enrolment will lower the risk of postoperative complications; however, this assumption is not supported by evidence from randomised controlled trials (RCTs).

Several studies were recently conducted in China to investigate the effectiveness of PTAS for patients with ICAS. $^{10} 1516$ The first one was a single-centre randomised trial, the results of which demonstrated that PTAS is as effective as medical treatment. ${ }^{16}$ The result of the second study, a multicentre registry study, showed that the incidence of stroke, transient ischaemic attack (TIA) and death in 30 days after stenting was $4.3 \%$, which is lower than the incidence in the SAMMPRIS trial. ${ }^{17}$ The third study was a retrospective study. This study showed that the incidence of stroke was higher when PTAS was performed by inexperienced surgeons, which is different from the result of a subgroup analysis of the SAMMPLIS trial. Moreover, the 1-year cumulative stroke rate, reaching $9.5 \%$, was still higher than expected. ${ }^{18}$ Taken together, the results of these studies indicate that further investigation of the effectiveness and safety of adding PTAS to background medication is still needed. Our primary aim is to compare medication treatment (MT) plus PTAS with MT alone in the treatment of patients with symptomatic ICAS.

\section{METHODS}

\section{Study design}

From 2016 to 2019, we will conduct a RCT to clarify whether PTAS plus MT is superior to MT in reducing the incidence of cerebrovascular events in patients with ICAS. In this RCT, patients with ICAS will be randomly assigned to a MT plus PTAS (MT+PTAS) group or a MT group in a 1:1 ratio. Patients in the MT+PTAS group will receive PTAS when alarm symptoms are relieved after MT treatment; patients in the MT group will receive MT alone. The MT treatment will be initiated immediately after randomisation. All patients will be followed up at 30 days, 3 and 12 months after randomisation. In the follow-up visits, the incidence of death or strokes in the stenotic vessels (responsible vessels causing alarm symptoms), the rate of patients with residual stenosis $<30 \%$, the incidence of recurrent ischaemic stroke or in-stent restenosis, the Chinese version of the modified Rankin Scale (mRS), and the Chinese version of Stroke-specific Quality of Life (CSQoL) will be assessed. Figure 1 shows a flowchart of the study. Figure 2 shows the schedule of enrolment, intervention and assessment.

\section{Participants}

We will recruit patients with at least three TIAs or a previous ischaemic stroke and patients with an ICAS stenosis with luminal narrowing from $70 \%$ to $99 \%$ detected by catheter angiography. Any patient who reports an ischaemic stroke in the 3 weeks before enrolment will be excluded, since ischaemic stroke may be a consequence of haemorrhagic transformation. Detailed inclusion and exclusion criteria of this trial are provided in boxes 1 and 2. The three hospitals are invited to participate under the condition that each of them has more than 100 intracranial stenting operations per year, since study centres with low-volume enrolment showed a higher incidence of stroke or death than those with highvolume enrolment. ${ }^{12}$ In addition, the experience of a physician performing PTAS and postoperative complications are also important factors that may influence the outcome of patients receiving PTAS. ${ }^{10}$ Therefore, a physician who takes part in this study should have a minimum of 25 cases for carotid endarterectomy and 50 cases for carotid artery stenting. ${ }^{19}$ The methods used to control postoperative complications are described in the intervention and comparison section. We will exclude any patient with a vessel size smaller than $2.5 \mathrm{~mm}$, aged over 70 years, or with a cerebral infarction owing to perforator artery occlusion because these patients are inclined to have strokes. ${ }^{8} 1314$ The vessel size will be determined with intracranial angiography. Whether a patient has a perforator stroke will be determined by

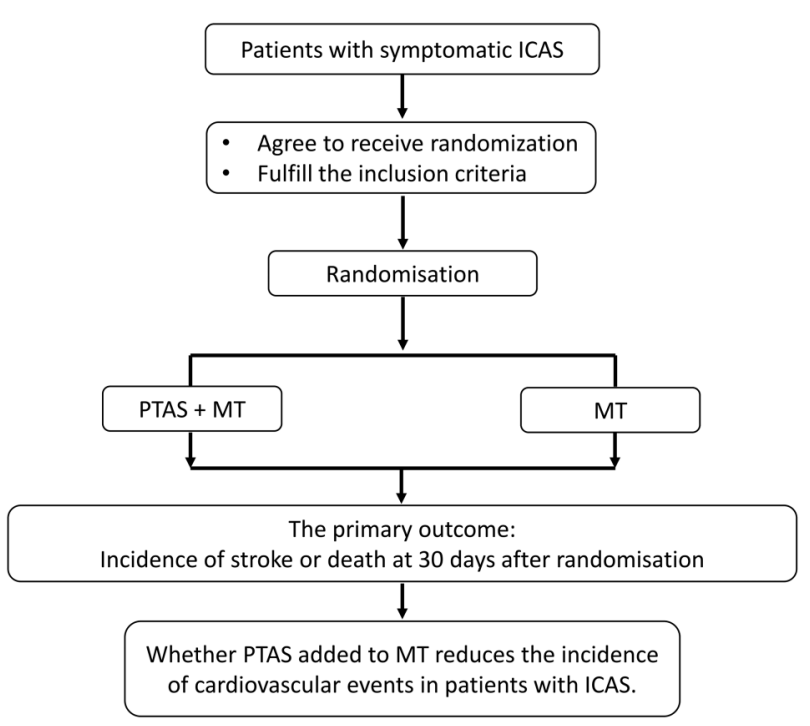

Figure 1 Flow chart of the study. ICAS, intracranial artery stenosis. PTAS, percutaneous transluminal angioplasty and stenting. MT, medication treatment. 


\begin{tabular}{|c|c|c|c|c|c|}
\hline \multirow{3}{*}{ Items } & \multicolumn{5}{|c|}{ Study Period } \\
\hline & \multirow{2}{*}{$\begin{array}{c}\text { Enrolment } \\
\text { Baseline }\end{array}$} & \multirow{2}{*}{$\begin{array}{c}\text { Allocation } \\
\text { Randomisation }\end{array}$} & \multirow{2}{*}{$\begin{array}{l}\text { Treatment } \\
30 \text { days }\end{array}$} & \multicolumn{2}{|c|}{ Follow-up } \\
\hline & & & & 3 months & 12 months \\
\hline \multicolumn{6}{|l|}{ Enrolment } \\
\hline $\begin{array}{l}\text { Eligibility } \\
\text { screen }\end{array}$ & $x$ & & & & \\
\hline $\begin{array}{l}\text { Informed } \\
\text { consent }\end{array}$ & $x$ & & & & \\
\hline Allocation & & $x$ & & & \\
\hline \multicolumn{6}{|l|}{ Intervention } \\
\hline MT+PTAS & & & $x$ & & \\
\hline MT & & & $x$ & & \\
\hline \multicolumn{6}{|l|}{ Outcome } \\
\hline \multicolumn{6}{|l|}{ Measures } \\
\hline Incidence of & & & x & $x$ & $x$ \\
\hline stroke or death & & & & & \\
\hline $\begin{array}{l}\text { Incidence of } \\
\text { ischaemic stroke } \\
\text { in the territory } \\
\text { stenosis arteries }\end{array}$ & & & $x$ & $x$ & $x$ \\
\hline $\begin{array}{l}\text { Incidence of } \\
\text { in-stent } \\
\text { restenosis }\end{array}$ & & & $x$ & $x$ & $x$ \\
\hline $\mathrm{mRS}$ & & $x$ & $x$ & $x$ & $x$ \\
\hline CSQoL & & $x$ & $x$ & $x$ & $x$ \\
\hline
\end{tabular}

Figure 2 Schedule of enrolment, intervention and assessments. MT, medication treatment. PTAS, percutaneous transluminal angioplasty and stenting. mRS, the Chinese version of the modified Rankin Scale. CSQoL, the Chinese version of Stroke-specific Quality of Life.

magnetic resonance (MR) perfusion. If possible, we will also use MR angiography to locate stenosis in perforator arteries. We will use two external markers of known size to calculate the vessel size. ${ }^{20}$ The markers will be attached to the temporal-frontal regions of a patient's head, and the magnification factor at the location of the target vessel will be computed by interpolation between the magnification factors acquired from the two markers. Cerebral hypoperfusion caused by ICAS will be assessed with transcranial Doppler measuring cerebral blood flow (CBF) velocity; a low CBF velocity indicates hypoperfusion. ${ }^{21}$ We will also use MR perfusion to confirm whether a patient has intracranial hypoperfusion.

\section{Recruitment of participants}

With the help of voluntary workers, we will post advertisements in communities. Emails will be sent to potential candidates who had examinations or treatments in the three hospitals. We will also use social media (advertisements in newspapers or local television channels) or online communication to reach potential candidates. Leaflets will be disseminated in the three hospitals; placards and signs will be posted in the outpatient and

\section{Box 1 Inclusion criteria}

1. Patients aged from 40 to 70 years

2. Patients with at least three transient ischaemic attacks whose condition failed to respond to a maximal dose of aspirin (defined as $300 \mathrm{mg}$ per day) or patients with an ischaemic stroke within 1 year owing to a $70-99 \%$ stenosis in an intracranial carotid artery or middle cerebral artery

3. Patients presenting cerebral hypoperfusion in the territory of target vessels, which is determined by MR perfusion in 14 days before stenting

4. Patients with at least one stenosis in the target vessel, a stenotic length $\leq 15 \mathrm{~mm}$, and a vessel size $>2.5 \mathrm{~mm}$

5. Patients who understand the purpose of this study and provide informed consent

*The vessel size will be determined with intracranial angiography. We will use two external markers of known size to calculate the vessel size. The markers will be attached to the temporal-frontal regions of a patient's head, and the magnification factor at the location of the target vessel will be computed by interpolation between the magnification factors acquired from the two markers. ICAS, intracranial atherosclerosis; MR, magnetic resonance.

\section{Box 2 Exclusion criteria}

1. Patients cannot receive general anaesthesia

2. Patients cannot receive angiographic assessment

3. A cerebral infarction is caused by perforator occlusion (determined by MR perfusion), which is defined as a basal ganglia infarction

4. CT or MRI scans show massive cerebral infarction (beyond half of the MCA territory), intracranial haemorrhage, epidural or subdural haemorrhage, and intracranial brain tumour

5. Patients are at high risk (leading to a stroke or death) in delivering a stent to a stenotic lesion

6. There is a previous stent or angioplasty in the target lesion

7. Patients show progressive neurological signs in the 24 hours before enrolment

8. Patients present with a haemorrhagic infarction in the 14 days before enrolment

9. There is a cardiac source of embolus in a patient

10. Patients have received thrombolytic therapy in the 24 hours before enrolment

11. Patients present with intraluminal thrombus proximal to or at the target lesion

12. Patients have been diagnosed with myocardial infarction in the 30 days before enrolment

13. Patients have been diagnosed with any of the following nonatherosclerotic lesions: arterial dissection, moya-moya disease, vasculitic disease, any intracranial infection, any intracranial stenosis associated with cerebrospinal fluid pleocytosis, radiation-induced vasculopathy, fibromuscular dysplasia; sickle cell disease, neurofibromatosis, benign angiopathy of central nervous system, suspected vasospastic process, or suspected embolus

14. Patients have known contraindications to aspirin and clopidogrel administration

15. Patients have a modified Rankin scale score $\geq 3$

MCA, middle cerebral artery; MRI, magnetic resonance imaging. 
inpatient settings. Patients who are interested in this study will be referred to research assistants in the three hospitals, and the assistants will discuss the aim and design of this trial with the patients.

\section{Randomisation and concealment}

A randomisation sequence will be generated with permuting blocks by the Clinical Evaluation Center in Fujian Medical University (CECFMU), stratified according to the degree of stenosis $(70-79 \%, 80-89 \%$ and $90-$ $99 \%$ ). The sequence will be concealed by a staff member in the evaluation centre. When an eligible participant is recruited, an investigator will acquire the randomisation code and group assignment through web-based registration, phone call or mobile phone text. The member of staff who conceals the sequence will respond to the request and record the randomisation message.

\section{Intervention and comparison}

Patients in both groups will receive background MT treatment, which includes dual-antiplatelet treatment and intensive risk management. The dual-antiplatelet treatment includes administration of aspirin (100 mg per day) and clopidogrel (75 $\mathrm{mg}$ per day). A previous study showed that combined use of aspirin and clopidogrel for 90 days was the most effective treatment for patients with ICAS, ${ }^{22}$ so we will ask the patients to stick to the dual-antiplatelet treatment for 90 days. Additional medical treatment will be administered to achieve a target systolic blood pressure under $140 \mathrm{~mm} \mathrm{Hg}$ and a HbA1c level under $6.5 \%$. Atorvastatin will be started at a loading dose of $10 \mathrm{mg}$ per day, and the dose will increase until the target level of low-density lipoprotein (LDL-C) is reached (LDL-C $<100 \mathrm{mg} / \mathrm{dL}$ ). Interventions for lowering the risk of cerebrovascular and cardiovascular diseases will also be used, such as lifestyle modification and smoking cessation.

Patients in the MT+PTAS group will receive the same MT treatment. When an emergent stenting operation is needed, a loading dose of $300 \mathrm{mg}$ clopidogrel will be given. Patients in the MT+PTAS group will receive PTAS when alarm symptoms are relieved after the MT treatment.

A standard protocol of the PTAS procedure will be developed, and the procedure will be performed by qualified physicians in each participating centre. The PTAS procedure will be developed in three parts: before, during and after the PTAS. Before the PTAS, the condition of arteria femoralis will be examined through colour Doppler ultrasound, since we will perform transfemoral puncture to get cerebral angiography and to start PTAS. Amid the PTAS procedure, fentanyl 20-50 $\mu \mathrm{g}$ will be used intravenously for $20-30 \mathrm{~min}$ to achieve general analgesia. An introducer needle will be inserted into the femoral artery. A short sheath $(10-13 \mathrm{~cm}$ in length) is placed in the opening to keep the artery open and control bleeding. The inner diameter of the sheath will be $4-10 \mathrm{~F}$; the outer diameter will be $1.5-2.0 \mathrm{~F}$ larger. A guide catheter will be pushed through the sheath; dyes will be injected through the guide catheter into intracranial arteries to locate the stenosis. After locating the X-ray image, an operating neurologist will select the type of balloon catheter $(0.6-1.6 \mathrm{~mm}$ in inner diameter) and guidewire (150-200 cm in length). When the guidewire reaches the stenosis, we will push it through and insert a hollow-tipped balloon catheter, using the guidewire as a pathway to the site of the stenosis. After the balloon is pushed inside the stenosis, we will inflate the balloon to compress the blockage and insert a stent (the Wingspan stent system, Stryker Neurovascular, Mountain View, California, USA) to keep the artery wall expanded. The stent diameter will be equal to or the next size up from the largest vessel diameter. For example, a $4.0 \mathrm{~mm}$ stent will be implanted in a $4.0 \mathrm{~mm}$ vessel size, while a $4.5 \mathrm{~mm}$ stent will be placed in a $4.1 \mathrm{~mm}$ vessel size. The Wingspan system is suitable for lesions in the distal internal carotid and middle cerebral arteries, ${ }^{23}$ which are difficult to access with balloonmounted stents, such as the Neurolink system. Additionally, the Wingspan system may provide additional benefit for patients with ICAS whose condition is refractory to medical therapy. The whole procedure will last for about 1 hour. After the PTAS, the patients will be transferred to the recovery area to receive routine care. To reduce the risk of postoperative complications, first, we will select the Wingspan system which is related to a lower risk of complications. ${ }^{24}$ Second, postoperative heparin is associated with an increased rate of intracerebral haemorrhage. ${ }^{25}$ So we will stop heparin use 6 hours prior to PTAS and 48 hours after PTAS. ${ }^{26}$ Third, PTAS performed within 24 hours after onset of an index event is associated with increased risk of complications. ${ }^{27}$ Therefore, we will perform PTAS after patients report relief of alarm symptoms defined as sudden weakness or numbness. We will record the time to achieve symptom relief or the duration of symptoms leading to symptom resolution, which will be included in the final analysis.

\section{Outcome measures}

The primary outcome is the incidence of stroke in a target vessel (including haemorrhagic or ischaemic stroke) or death at 30 days after randomisation. An ischaemic stroke is defined as a new focal neurological deficit of sudden onset, lasting at least 24 hours. The stroke is not caused by haemorrhage determined by cranial CT or MRI scan. The ischaemic stroke will be classified as inside or outside of the territory of target vessels. Symptomatic brain haemorrhage is defined as parenchymal, subarachnoid or intraventricular haemorrhage detected by CT or MRI scan, and the haemorrhage is associated with seizures or new neurological symptoms or physical signs (headache, focal neurological deficits, or change in consciousness level) lasting at least 24 hours. The brain haemorrhage will be regarded as achieving primary outcome if it occurs within 30 days after PTAS. 
The secondary outcomes are the incidence of recurrent ischaemic stroke in the stenting-involved vascular territory at 30 days, 3 and 12 months after randomisation, the rate of patients with residual stenosis $<30 \%$ at 3 and 12 months after randomisation, the incidence of in-stent restenosis at 12 months after randomisation, the incidence of cerebral parenchymal, subarachnoid or intraventricular haemorrhage at 30 days, 3 and 12 months after randomisation, the incidence of myocardial infarction or major non-stroke haemorrhage (epidural or subdural haemorrhage or haemorrhage in major organs) at 30 days after randomisation, the Chinese version of National Institutes of Health Stroke Scale (C-NIHSS), ${ }^{28}$ the $\mathrm{mRS}^{29}{ }^{29}$ and the $\mathrm{CSQOL}^{30}$ at 30 days, 3 and 12 months after randomisation. To assess whether residual stenosis or in-stent restenosis exists, we will use the trans-femoral cerebral angiography.

We will diagnose a patient with myocardial infarction if the patient has any of the following conditions: new ST segment depression or elevation $>1 \mathrm{~mm}$ in more than two contiguous leads shown by electrocardiograph, significant increase in creatine kinase or cardiac troponins T and I within 3 to 12 from onset of chest pain. ${ }^{31}$

\section{Data collection and monitoring}

A data managing and monitoring board will be set up to supervise data acquisition and entry. Adverse events or unintended effects of PTAS plus MT will first be collected by physicians who are in charge, then the physicians will call the data monitoring centre within 24 hours. Collected data will be entered into electronic case report forms (eCRFs) with double entry and logic validation. The eCRFs will be uploaded to a central server. The principle investigator (Hang Lin) will have full access to the data. A clinical associate independent from the investigators and sponsors will audit the trial conduct every month after the trial starts. The clinical associate will write monthly reports on recruitment of participants, compliance of the participants to assigned treatment, and whether the case report forms are correctly filled in.

\section{Sample size calculation}

A retrospective study showed that the 1-year rate of stroke was $9.5 \%$ for patients with ICAS after PTAS, ${ }^{18}$ and the 1-year rate of stroke after dual antiplatelet therapy (aspirin and clopidogrel) is $17.2 \%$ in the SAMMPRIS trial. $^{7}$ Therefore, we anticipate a difference of $7.7 \%$ between the two arms. Power calculation (80\%) for sample size is performed based on a two-sided test with a significance level of $5 \%$. The sample size in each group is estimated to be 245 . If the rate of patients lost to follow-up or early withdrawal is set at $10 \%$, a total of 539 participants will be needed.

\section{Statistical analysis}

All analyses will be performed on the basis of intention-to-treat population. Missing values will be handled with multiple imputation (continuous variables) or last-observation-carried-forward method (category variables). In the multiple imputation, we will assume that the missing data follow a linear distribution, and we will run the simulation in five different calculations with the Mice package in $\mathrm{R}$ software (http://www.r-project. org, version 3.1.0). We hypothesise ' $\mathrm{H}_{0}$ : $\mathrm{PTAS}+\mathrm{MT}=\mathrm{MT}$; $\mathrm{H}_{1}$ : PTAS+MT $\neq$ MT', and a Cox proportional hazards model will be used to test the hypothesis. The Cox model will be used to calculate the HR of the primary outcome at 30 days after randomisation and test the statistical difference between the two groups. The Cox model will be adjusted with study centres and vessel size; the model will include treatment, treatment by time interaction, factors, and the treatment by factors interaction. The cumulative probability of the primary outcome at 12 months after randomisation will be also be compared between the two groups in the Cox model. The secondary outcomes will be analysed with the same Cox model.

To ensure the robustness of the results, two sensitivity analyses will be used to generate data for patients withdrawing or being lost to follow-up. First, only patients in the PTAS group will be considered to have a primary outcome in the censored data. Second, only patients in the medical group will be considered to have a primary outcome in the censored data. All reported $\mathrm{p}$ values are two sided without adjustment for multiple testing. We will use SPSS (IBM Corp, Armonk, New York, V.20.0) and $\mathrm{R}$ software for all statistical analyses.

\section{ETHICS AND DISSEMINATION}

The study proposal and study materials were reviewed by the institutional review boards (IRBs) in the three participating centres. All the members in the IRBs approved the trial protocol (Fuzhou General Hospital of Nanjing Command, reference number FZ20160003; the 180th Hospital of the People's Liberation Army, reference number: 180PLA20160101; the 476th Hospital of the People's Liberation Army, reference number: 476PLA2016007). The study is registered at clinicaltrials. gov (NCT02689037). When significant modification occurs, we will inform the sponsor, all investigators, participants, and the trial registry. Research assistants in each participating centre will obtain informed consent from each participant before they enter the study using standardised consent forms. Contact details for support organisations will be given to participants in case they need information to ensure the safety of the participants.

Personal information about the enrolled participants will be safely and confidentially kept in the three hospitals. The results from this trial will be submitted to a peer-reviewed journal for publication. The anonymised individual patient data will be shared on request.

\section{DISCUSSION}

This study is anticipated to finish at the end of the year 2019 and the results will clarify whether MT plus PTAS is 
superior to MT alone for decreasing the incidence of stroke or death related to symptomatic ICAS. This study will include patients with TIA or stroke in the same cohort, which may raise some concern that there is a difference in the 1 year rate of stroke or death in these two groups of patients. There are limited data on the difference between the 1 year stroke rate in patients with TIA and those with non-disabling stroke. The WASID study included patients with TIA and stroke who were diagnosed with stenosis in major intracranial arteries (carotid, middle cerebral, vertebral or basilar). ${ }^{32}$ Moreover, most subsequent strokes (we defined as recurrent strokes in our study) in patients with symptomatic intracranial artery are in the same territory. ${ }^{4}$ So the difference in vessel pathology between patients with TIA and stroke will not influence the risk of strokes in the territory of stenotic arteries. In this RCT, we will adopt an endovascular treatment protocol combining balloon angioplasty with stenting. Angioplasty alone is as effective as carotid endarterectomy. ${ }^{33}$ Angioplasty has several advantages. It can be done with local anaesthesia and mild sedation; it has a short recovery period; and it reduces cerebrovascular events. However, despite plenty of advantages, its major disadvantage is that the responsible artery may close again. So stenting after balloon angioplasty is becoming a standard protocol of endovascular treatment in intracranial arteries. No study has compared PTAS with balloon angioplasty alone in preventing strokes in patients with ICAS, but there is a study showing additional benefit of stenting added to percutaneous transluminal coronary angioplasty. ${ }^{34}$ So we assumed that patients with ICAS will benefit the most from PTAS treatment. Compared with the SAMPPRIS trial, our study will avoid inclusion of patients with perforator stroke. These patients benefit little from additional PTAS according to previous studies, and studies showing a high incidence of postoperative complications may be related to inclusion of these patients. Second, our study will only allow centres with high-volume patient enrolment to participate. According to a subgroup analysis of the SAMPPRIS trial, centres with lowvolume enrolment reported more postoperative complications. ${ }^{12}$ Third, we will include patients with recurrent TIA (defined as having at least three attacks and not responding to a maximal dose of aspirin), which is not clearly defined in the SAMMPRIS trial. Compared with the VISSIT trial, our study has two advantages. First, we have a larger sample size, which increases study power. Second, we will exclude patients with ICAS in the vertebral or basilar artery to focus on anterior circulation. This design is advocated by neurologists who insist that patients with ICAS in anterior circulation benefit more than those with ICAS in posterior circulation. We defined the primary outcome as the incidence of strokes in the territory of arteries with stenosis (target vessels) or death at 30 days after randomisation instead of the incidence of any stroke or death, because it is impossible for strokes in non-target vessels to be caused by stenosis in target vessels, so counting strokes in non-target vessels may underestimate the benefit of adding PTAS to medical treatment.

The strength of the study is that we will use an improved surgical protocol in PTAS treatment, exclude patients with perforator syndrome, and admit participating centres with high-volume enrolment, all of which will significantly reduce the postoperative complications that are contributors to a high incidence of stroke or death. The results of this study will be disseminated through journal articles and conference presentations.

Contributors X-PC and HL conceived and designed this study. ML, J-SM W-QH, M-LF, HL and J-YF will collect the data. F-FS will control the quality of trial. J-XY will analyse and interpret the data. X-PC and $\mathrm{HL}$ drafted this protocol. All the authors have reviewed and approved the publication of this study protocol.

Funding This study is financially supported by Health Research Fund from People's Liberation Army (No. 12MA100). The study sponsor and funders play no role in study design, collection, management, analysis, and interpretation of data, writing of the report and the decision to submit the report for publication.

Competing interests None declared.

\section{Patient consent Obtained.}

Ethics approval Fuzhou General Hospital of Nanjing Command, reference number: FZ20160003; the 180th Hospital of the People's Liberation Army, reference number: 180PLA20160101; the 476th Hospital of the People's Liberation Army, reference number: 476PLA2016007.

Provenance and peer review Not commissioned; externally peer reviewed.

Data sharing statement This is a study protocol. We would like to share data through the public web to all the investigators who are interested in this topic.

Open Access This is an Open Access article distributed in accordance with the Creative Commons Attribution Non Commercial (CC BY-NC 4.0) license, which permits others to distribute, remix, adapt, build upon this work noncommercially, and license their derivative works on different terms, provided the original work is properly cited and the use is non-commercial. See: http:// creativecommons.org/licenses/by-nc/4.0/

\section{REFERENCES}

1. Bos D, van der Rijk MJ, Geeraedts TE, et al. Intracranial carotid artery atherosclerosis prevalence and risk factors in the general population. Stroke 2012;43:1878-84.

2. Ritz K, Denswil NP, Stam OC, et al. Cause and mechanisms of intracranial atherosclerosis. Circulation 2014;130:1407-14.

3. Holmstedt CA, Turan TN, Chimowitz MI. Atherosclerotic intracranial arterial stenosis: risk factors, diagnosis, and treatment. Lancet Neurol 2013;12:1106-14.

4. Kasner SE, Chimowitz MI, Lynn MJ, et al. Predictors of ischemic stroke in the territory of a symptomatic intracranial arterial stenosis. Circulation 2006;113:555-63.

5. Wang Y, Zhao X, Liu L, et al. Prevalence and outcomes of symptomatic intracranial large artery stenoses and occlusions in China: the Chinese Intracranial Atherosclerosis (CICAS) Study. Stroke 2014;45:663-9.

6. Chimowitz MI, Lynn MJ, Derdeyn CP, et al. Stenting versus aggressive medical therapy for intracranial arterial atenosis. $N$ Engl J Med 2011;365:993-1003.

7. Derdeyn CP, Chimowitz MI, Lynn MJ, et al. Aggressive Medical Treatment with or without Stenting in High-risk Patients with Intracranial Artery Stenosis (SAMMPRIS): the final results of a randomised trial. Lancet 2014;383:333-41.

8. Abou-Chebl A, Steinmetz $H$. Critique of 'Stenting versus aggressive medical therapy for intracranial arterial stenosis' by Chimowitz et al in the New England Journal of Medicine. Stroke 2012;43:616-20. 
9. Tian L, Yue X, Xi G, et al. Multiple intracranial arterial stenosis influences the long-term prognosis of symptomatic middle cerebral artery occlusion. BMC Neurol 2015;15:68.

10. Gao P, Zhao Z, Wang D, et al. China Angioplasty and Stenting for Symptomatic Intracranial Severe Stenosis (CASSISS): a new, prospective, multicenter, randomized controlled trial in China. Interv Neuroradiol 2015;21:196-204.

11. Qureshi Al, Al-Senani FM, Husain S, et al. Intracranial angioplasty and stent placement after stenting and aggressive medical management for preventing recurrent stroke in intracranial stenosis (SAMMPRIS) trial: present state and future considerations. J Neuroimaging 2012;22:1-13.

12. Derdeyn CP, Fiorella D, Lynn MJ, et al. Impact of operator and site experience on outcomes after angioplasty and stenting in the SAMMPRIS trial. $J$ Neurointer Surg 2013:5:528-33.

13. Derdeyn CP, Fiorella D, Lynn MJ, et al. Mechanisms of stroke after intracranial angioplasty and stenting in the SAMMPRIS trial. Neurosurgery 2013;72:777-95; discussion 95.

14. Fiorella D, Derdeyn CP, Lynn MJ, et al. Detailed analysis of periprocedural strokes in patients undergoing intracranial stenting in Stenting and Aggressive Medical Management for Preventing Recurrent Stroke in Intracranial Stenosis (SAMMPRIS). Stroke 2012;43:2682-8.

15. Wang $\mathrm{Y}$, Miao Z, Wang $\mathrm{Y}$, et al. Protocol for a prospective, multicentre registry study of stenting for symptomatic intracranial artery stenosis in China. BMJ Open 2014;4:e005175.

16. Miao Z, Jiang $\mathrm{L}, \mathrm{Wu} \mathrm{H}$, et al. Randomized controlled trial of symptomatic middle cerebral artery stenosis: endovascular versus medical therapy in a Chinese population. Stroke 2012;43:3284-90.

17. Miao Z, Zhang Y, Shuai J, et al. Thirty-day outcome of a multicenter registry study of stenting for symptomatic intracranial artery stenosis in China. Stroke 2015;46:2822-9.

18. Li TX, Gao BL, Cai DY, et al. Wingspan stenting for severe symptomatic intracranial atherosclerotic stenosis in 433 patients treated at a Single Medical Center. PLOS ONE 2015;10:e0139377.

19. Hobson RW IInd, Howard VJ, Roubin GS, et al. Credentialing of surgeons as interventionalists for carotid artery stenting: experience from the lead-in phase of CREST. $J$ Vasc Surg 2004;40:952-7.

20. Divani AA, Tholany CR, Siddiqui AU, et al. Comparison of 1- and 2-marker techniques for calculating system magnification factor for angiographic measurement of intracranial vessels. J Neuroimaging 2005;15:356-61.
21. Ruitenberg A, den Heijer T, Bakker SLM, et al. Cerebral hypoperfusion and clinical onset of dementia: the Rotterdam study. Ann Neurol 2005;57:789-94.

22. Wang $Y$, Wang $Y$, Zhao $X$, et al. Clopidogrel with aspirin in acute minor stroke or transient ischemic attack. N Eng J Med 2013;369:11-9.

23. Killer M, Ladurner G, Kunz AB, et al. Current endovascular treatment of acute stroke and future aspects. Drug Discov Today 2010;15:640-7.

24. Abou-Chebl A. Intracranial stenting with Wingspan: still awaiting a safe landing. Stroke 2011;42:1809-11.

25. Meyers PM, Higashida RT, Phatouros CC, et al. Cerebral hyperperfusion syndrome after percutaneous transluminal stenting of the craniocervical arteries. Neurosurgery 2000;47:335-45.

26. Kaufmann TJ, Huston JIII, Mandrekar JN, et al. Complications of diagnostic cerebral angiography: evaluation of 19826 consecutive patients 1. Radiology 2007;243:812-19.

27. Jiang WJ, Cheng-Ching E, Abou-Chebl A, et al. Multicenter analysis of stenting in symptomatic intracranial atherosclerosis. Neurosurgery 2012;70:25-31.

28. Sun TK, Chiu SC, Yeh SH, et al. Assessing reliability and validity of the Chinese version of the stroke scale: Scale development. Int J Nurs Stud 2006;43:457-63.

29. Yuan JL, Bruno A, Li T, et al. Replication and extension of the simplified modified Rankin Scale in 150 Chinese stroke patients. Eur Neurol 2012;67:206-10.

30. Wang YL, Ma JG, Li JT. The study on reliability, validity and responsiveness of the Chinese version of Stroke-specific Quality of Life. Chin J Geriatr Cardiovasc Cerebrovasc Dis 2003;6:391-4.

31. Halim SA, Mulgund J, Chen AY, et al. Use of guidelines-recommended management and outcomes among women and men with low-level troponin elevation: insights from CRUSADE. Circ Cardiovasc Qual Outcomes 2009;2:199-206.

32. Chimowitz Ml, Lynn MJ, Howlett-Smith $\mathrm{H}$, et al. Comparison of warfarin and aspirin for symptomatic intracranial arterial stenosis. N Engl J Med 2005;352:1305-16.

33. Brown M, Rogers J, Bland J. Endovascular versus surgical treatment in patients with carotid stenosis in the Carotid and Vertebral Artery Transluminal Angioplasty Study (CAVATAS): a randomised trial. Lancet 2001;357:1729-37.

34. Mehran R, Dangas G, Abizaid A, et al. Treatment of focal in-stent restenosis with balloon angioplasty alone versus stenting: short-and long-term results. Am Heart J 2001;141:610-14. 